\title{
A Dynamic Programming Technique for Optimizing Dissimilarity-Based Classifiers ${ }^{\star}$
}

\author{
Sang-Woon $\mathrm{Kim}^{1}$ and Jian $\mathrm{Gao}^{2}$ \\ ${ }^{1}$ Senior Member, IEEE. Address: Dept. of Computer Science and Engineering, Myongji \\ University, Yongin, 449-728 South Korea \\ kimsw@mju.ac.kr \\ ${ }^{2}$ Address: Dept. of Computer Science and Engineering, Myongji University, Yongin, 449-728 \\ South Korea \\ marsgao@mju.ac.kr
}

\begin{abstract}
The aim of this paper is to present a dissimilarity measure strategy by which a new philosophy for pattern classification pertaining to dissimilaritybased classifiers (DBCs) can be efficiently implemented. Proposed by Duin and his co-authors, DBCs are a way of defining classifiers among classes; they are not based on the feature measurements of individual patterns, but rather on a suitable dissimilarity measure among the patterns. The problem with this strategy is that we need to measure the inter-pattern dissimilarities for all the training samples to ensure there is no zero distance between objects of different classes. Consequently, the classes do not overlap, and therefore, the lower error bound is zero. In image classification tasks, such as face recognition, one of the most intractable problems is the distortion and lack of information caused by the differences in face directions and sizes. To overcome the above problem, in this paper, we propose a new method of measuring the dissimilarity distance between two images of an object when the images have different directions and sizes and there is no direct feature correspondence. In the proposed method, a dynamic programming technique, such as dynamic time warping, is used to overcome the limitation of one-to-one mapping. Furthermore, when determining the matching templates of two images in dynamic time warping, we use a correlation coefficient-based method. With this method, we can find an optimal warping path by surveying the images in a one-dimensional or two-dimensional way (that is, with vertical-only scanning or vertical-horizontal scanning). Our experimental results demonstrate that the proposed mechanism can improve the classification accuracy of conventional approaches for an artificial data set and two real-life benchmark databases.
\end{abstract}

\section{Introduction}

One of the most recent and novel developments in the field of statistical pattern recognition (PR) [1] is the concept of dissimilarity-based classifiers (DBCs) proposed by Duin and his co-authors (see [2] and [3]). Philosophically, the motivation for DBCs is as follows: If we assume that similar objects can be grouped together to form a class, the "class" is nothing more than a set of those similar objects [4]. On the basis of this idea, Duin and his colleagues argue that the notion of proximity (similarity or dissimilarity) is

\footnotetext{
^ This work was supported by the Korea Research Foundation Grant (KRF-2007-313-D00714).
} 
actually more fundamental than that of a feature or a class. Indeed, it is more likely that the brain uses an intuitive DBC-based methodology rather than taking measurements, inverting matrices, and so on. Thus, DBCs are a way of defining classifiers among the classes; and the process is not based on the feature measurements of individual patterns, but rather on a suitable dissimilarity measure among the individual patterns [4]. The advantage of this method is that because it does not operate on class-conditional distributions, the accuracy can exceed the Bayes error bound; moreover, the method actually attempts to attain a zero-error bound 1], - which, in our opinion, is remarkable. Another salient advantage of such a paradigm is that it can avoid the problems associated with feature spaces, such as the "curse of dimensionality" [1], and the issue of estimating a large number of parameters.

In spite of the advantages of DBCs, several problems need to be resolved when they are applied to particular tasks, such as face recognition [5], [6]. The three major questions we encountered when designing the DBCs concern the selection of prototype subsets [3], [4], [7], [8]; the measurement of dissimilarities between object samples [9], [10], [11]; and the design of a classifier in the dissimilarity space [2], [12]. Various methods have been proposed in the literature [3], [4], [7] as a means of selecting a representative set of data that is both compact and capable of representing the entire data set. The details of these methods are omitted here because they are not directly related to the premise of our work. In the interest of completeness, however, we now attempt to explain the other two questions in the present paper.

Optimization of DBCs: There are a few ways by which the classification efficiency of DBCs can be optimized. The major task of this study is to deal with how the dissimilarity measure can be effectively computed. The reason we set this task as our goal comes from the necessity to measure the inter-pattern dissimilarities for all the training samples such that there is no zero distance between objects of different classes. Consequently, the classes do not overlap, and therefore, the lower error bound is zero. The application of DBCs for image classification, such as face recognition, requires accurate measurement of the dissimilarity between high-dimensional samples. Furthermore, in facial images, there are many kinds of variations based on such factors as pose (direction), facial expression, illumination, and distance. Thus, by simply measuring the differences in facial images for each class, we are unable to obtain a good representation. If a limited number of prototypes is available or the representational capability is insufficient to cover the possible variations of data, it is difficult to improve the performance of DBCs in the dissimilarity space.

To overcome this limitation and thereby improve the performance of DBCs, we propose another way of enriching the representational capability of dissimilarity measures. In particular, this goal can be achieved by using a dynamic programming technique 2 to

\footnotetext{
${ }^{1}$ The idea of the zero-error bound is based on the fact that dissimilarities may be defined such that there is no zero distance between objects of different classes. Consequently the classes do not overlap, and so the lower error bound is zero. We are grateful to Bob Duin for providing us with insight into this.

${ }^{2}$ Dynamic programming, in which the word programming means finding an acceptable plan of action, can deal with the local deformation or warping of the sequences by finding an optimal alignment (warping path) between two input sequences.
} 
overcome the limitation of one-to-one mapping; the technique involves a mathematical procedure of solving sequential decision problems in operations research. In PR applications, various dynamic programming techniques have been proposed in the literature to solve the problems of time alignment, normalization, localization, and so on [13], [14]. Dynamic programming techniques that could be used include dynamic template warping [15], dynamic space warping [16], and affine invariant dynamic time warping [17]. However, for the purpose of simplifying the classification problem, we used dynamic time warping (DTW) [14], one of the most well-known dynamic programming techniques. In DTW, one-to-many points (vectors) mapping is performed after two respective subregions have been determined from the corresponding images; the purpose of the mapping is to find the optimal alignment between two image samples that have different directions or sizes. For greater accuracy of the classification, we propose a correlation coefficient-based DTW method in which matching templates from the two images are found by using a correlation coefficient based method with vertical and horizontal scanning. In the proposed method, the dissimilarity is measured in two steps: first we adjust the faces by finding the best warping path, $w$, with the correlation coefficientbased DTW method; we then compute the dissimilarity distance between the adjusted faces with conventional measures.

Contributions of the Paper: Our study makes two modest contributions to this field:

1. Our experimental results are the first to optimize 3 dissimilarity-based classifiers by resorting to a DTW dynamic programming technique. Although our results apply only to a case of appearance-based face recognition, the proposed approach can also be used in other high-dimensional classification tasks, such as multimedia retrieval, bioinformatics, computer vision, and text classification.

2. The proposed approach combines general distance measures traditionally associated with dissimilarity-based classifiers with the ability of the dynamic programming technique to accommodate variations in direction and size. The dynamic programming technique is used to establish one-to-many (or many-to-one) mapping as well as oneto-one mapping between the deformable images of an object class.

To the best of the our knowledge, these two contributions are novel to the field of high-dimensional image classification. The remainder of the paper is organized as follows: In Section 2] after providing a brief introduction to the dissimilarity representation, we present an algorithm that improves the classification accuracy of DBCs by utilizing dissimilarity representation and a dynamic programming technique. In Section 3 we present the experimental results of an artificial image data set and two real-life benchmark databases. In Section 4, we present our concluding remarks.

\section{Optimizing DBCs with DTW}

Dissimilarity Representation: A dissimilarity representation of a set of samples, $T=$ $\left\{\boldsymbol{x}_{1}, \cdots, \boldsymbol{x}_{n}\right\} \in \Re^{d}$, is based on pairwise comparisons, and is expressed, for example, as an $n \times m$ dissimilarity matrix $D_{T, Y}[\cdot, \cdot]$, where $Y=\left\{\boldsymbol{y}_{1}, \cdots, \boldsymbol{y}_{m}\right\}$, a prototype set, is extracted from $T$, and the subscripts of $D$ represent the set of elements, on which the

\footnotetext{
${ }^{3}$ Technically, the word "optimizing" in this paper is used in the context of "improving".
} 
dissimilarities are evaluated. Thus, each entry $D_{T, Y}[i, j]$ corresponds to the dissimilarity between the pairs of objects $\left\langle\boldsymbol{x}_{i}, \boldsymbol{y}_{j}\right\rangle$, where $\boldsymbol{x}_{i} \in T$ and $\boldsymbol{y}_{j} \in Y$. Consequently, an object $\boldsymbol{x}_{i}$ is represented as a column vector as follows:

$$
\left[d\left(\boldsymbol{x}_{i}, \boldsymbol{y}_{1}\right), d\left(\boldsymbol{x}_{i}, \boldsymbol{y}_{2}\right), \cdots, d\left(\boldsymbol{x}_{i}, \boldsymbol{y}_{m}\right)\right]^{T}, 1 \leq i \leq n .
$$

Here, the dissimilarity matrix $D_{T, Y}[\cdot, \cdot]$ is defined as a dissimilarity space, on which the $d$-dimensional object, $\boldsymbol{x}$, given in the feature space, is represented as an $m$-dimensional vector $\delta(\boldsymbol{x}, Y)$, where if $\boldsymbol{x}=\boldsymbol{x}_{i}, \delta\left(\boldsymbol{x}_{i}, Y\right)$ is the $i$-th row of $D_{T, Y}[\cdot, \cdot]$. In this paper, the column vector $\delta(\boldsymbol{x}, Y)$ is simply denoted by $\delta_{Y}(\boldsymbol{x})$, where the latter is an $m$-dimensional vector, while $\boldsymbol{x}$ is $d$-dimensional 4 . Two factors to consider for a dissimilarity representation are to select a prototype subset from $T$, and to quantify the dissimilarity between two vectors. To do these things, various representative selection methods and dissimilarity measures have been proposed in [2], [3], and [4].

Fundamental to DBCs is the measure used to quantify the dissimilarity between two vector 5 . Extensive experiments on various dissimilarity measures are reported in [3] (see Table 2 of [3]). The measures tested in [3] essentially fall into three categories: (a) the city block, $L_{0.8}$, the Euclidean, and the max norm, which are special cases of the $L_{p}$ metric for $p=1,0.8,2$, and $\infty$, respectively; (b) the Hausdorff norm and its variants, which involve max-min computations; and (c) traditional pattern recognition norms, such as the template matching norm and the correlation norm. The details of these methods are omitted here in the interest of brevity, but can be found in [2].

Schema for the Proposed Solution: On the basis of what we have briefly discussed, a conventional dissimilarity-based classification is summarized in the following:

1. Select the representative set, $Y$, from the training set, $T$, by resorting to one of the prototype selection methods as described in [3] and [4].

2. Using Eq. (1), compute the dissimilarity matrix, $D_{T, Y}[\cdot, \cdot]$, in which each individual dissimilarity is computed on the basis of the measures described in [2] and [4].

3. For a testing sample, $\boldsymbol{z}$, compute a dissimilarity column vector, $\delta_{Y}(\boldsymbol{z})$, by using the same measure used in Step 2.

4. Achieve the classification by invoking a classifier built in the dissimilarity space, and by operating the classifier on the dissimilarity vector, $\delta_{Y}(\boldsymbol{z})$. For example, if the nearest neighbor classifier is used and $\delta_{Y}\left(\boldsymbol{x}_{i}\right)$ that lies closest to $\delta_{Y}(\boldsymbol{z})$ is determined, $\boldsymbol{z}$ is assigned to the class of $\boldsymbol{x}_{i}$.

From the above four steps, we can see that the performance of the DBCs relies heavily on how well the dissimilarity space, which is determined by the dissimilarity matrix, $D_{T, Y}[\cdot, \cdot]$, is constructed. To improve the performance, we need to ensure that the dissimilarity matrix is well designed. With regard to measuring the dissimilarity of the sample points, we prefer not to directly measure the dissimilarity from the object points; rather, we propose a way of using the DTW dynamic programming technique to adjust or scale the object samples. This measure of dissimilarity effectively serves

\footnotetext{
${ }^{4}$ From this perspective, it becomes clear that the dissimilarity representation can be considered as a way of solving the small sample size problem [12].

${ }^{5}$ The details of the binary, categorical, ordinal, symbolic and quantitative features are omitted here, but can be found in [3].
} 
as a new "feature" component in the dissimilarity space. In our method of optimizing DBCs, we use DTW6 to align two face images that have different directions and sizes. The rationale for the proposed method is conceptually explained in Figure 1

In Figure 1 we can see how DTW helps align two artificial faces, FaceA and FaceB. These faces have different directions: FaceA is facing left and FaceB is facing right. Thus, the two faces cannot be matched perfectly. To solve this problem, we propose a correlation coefficient-based DTW method. In this method, the dissimilarity is measured in an adjustment and a measurement step. In the adjustment step, the faces are aligned by matching the best warping path, $w$, with the correlation coefficients (see Figure 1). In the measurement step, the dissimilarity distance between the adjusted faces is then obtained with conventional measurements 7 . Theoretically, the stronger the linear relation between two vectors, the greater the similarity of their structures. On the basis of this idea, we use the right essence of the correlation coefficient as a measurement of the structural similarity between two face vectors 8 .

To obtain an optimal warping path for two objects, we first compute the correlation coefficient between two subregions (a subsequence of vectors) selected from the object matrices by scanning through them in a vertical-only way or a vertical-horizontal way. Figure 1 shows a plot that represents the process of the dynamic alignment of DTW and explains how the DTW aligns FaceA and FaceB to make them overlap completely. In Figure 1, the diagonal line of the box is an optimal warping path that records the mapping between the two faces. The mapping indicated as $M 1$ in the picture involves the mapping of "several" column vectors in FaceB to an "a" column vector in FaceA. In contrast, the mapping indicated as $M 2$ represents the mapping of an "a" column vector in FaceB to "several" column vectors in FaceA. On the basis of the alignment process (the optimal warping path) in Figure 1 we measured the dissimilarity of AdjustedFaceA and AdjustedFaceB, rather than FaceA and FaceB.

Optimized Dissimilarity-Based Classification: As mentioned earlier, a good representation cannot be obtained by simply measuring the dissimilarity of facial images for each class. To overcome this limitation, we used the DTW algorithm. The basic strategy of the technique is to solve the classification problem by first adjusting the faces, and then constructing a dissimilarity matrix with the correlation coefficient-based DTW method; finally, DBCs are designed on the dissimilarity space to reduce the classification error rates. The proposed approach, which is referred to as an Optimized Dissimilarity-Based Classifier (ODBC), is summarized in the following:

1. This step is the same as Step 1 in the conventional DBC.

2. Using Eq. (1), compute the dissimilarity matrix $D_{T, Y}[\cdot, \cdot]$, in which each individual dissimilarity is computed using the correlation coefficient-based DTW method, rather than using one of the measures described in [2] and [4].

3. This step is the same as Step 3 in the conventional DBC.

\footnotetext{
${ }^{6}$ The details of DTW is omitted here in the interest of compactness, but can be found in [14].

${ }^{7}$ An algorithmic description of these two steps will be provided in the unabridged version of this paper (journal paper).

${ }^{8}$ The correlation coefficient, $\rho_{x, y}$, between $x$ and $y$ with their expected values, $\mu_{x}$ and $\mu_{y}$, and standard deviations, $\sigma_{x}$ and $\sigma_{y}$, is defined as: $\rho_{x, y}=E\left(\left(x-\mu_{x}\right)\left(y-\mu_{y}\right)\right) / \sigma_{x} \sigma_{y}$, where $E$ is the operator for expected values.
} 


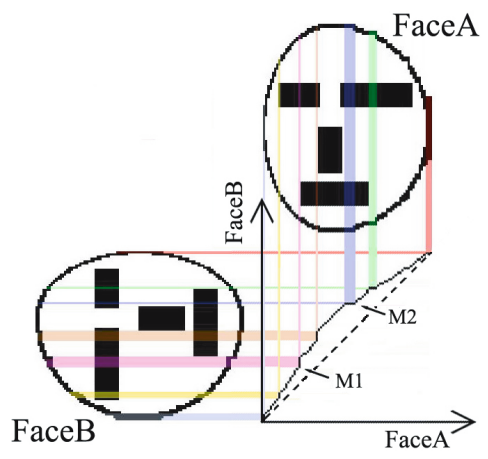

Fig. 1. The process of the DTW dynamic alignment. The DTW aligns FaceA and FaceB to make them overlap completely. The line in the picture is an optimal warping path, which records the mapping between the two faces. The details of the picture can be found in the text.

4. This step is the same as Step 4 in the conventional DBC.

As in the case of DBC, almost all the processing CPU-time of ODBC is utilized in executing Step 2. The time complexities and the space complexities of DBC and ODBC are, respectively, all the same as $O\left(d n^{2}+\gamma\right)$ and $O(n(n+d))$, where $\gamma$ is the time for doing classification with a classifier designed in the dissimilarity space.

\section{Experimental Results}

Experimental Data: The proposed method was tested and compared with conventional methods by conducting experiments on an artificial image data set and the two wellknown benchmark face databases, namely, "AT\&T",9 and "UMIST", 10 .

Figure 2 shows an artificial data set of two-dimensional images that the proposed method can handle. This data set was included in the experiments as a baseline data set. In Figure 2, ten copies of each picture are generated with random noise, where the intensity of the added random noise varies from $3 \%$ to $21 \%$ at increments of $2 \%$ per generation. Thus, the data set consists of 90 sample pictures per class and 180 sample pictures in total. In addition, the size of each binary picture is $64 \times 64$ pixels for a total dimensionality of 4096. The face database of AT\&T, formerly known as the ORL database of faces, consists of ten different images of 40 distinct subjects for a total of 400 images. The size of each image is $112 \times 92$ pixels for a total dimensionality of 10304 pixels. The UMIST repository is a multi-view face database, consisting of 564 images of 20 people, each covering a range of poses from profile to frontal views.

Experimental Method: All our experiments were performed with a "leave-one-out" strategy. To classify an image of an object, we removed that image from the training set and computed the dissimilarity matrix with the $n-1$ images. This process was repeated $n$ times for every sample, and a final result was obtained by averaging the results

\footnotetext{
${ }^{9} \mathrm{http}: / / \mathrm{www}$. uk.research.att.com/facedatabase.html

${ }^{10} \mathrm{http}: / /$ images.ee.umist.ac.uk/danny/database.html
} 


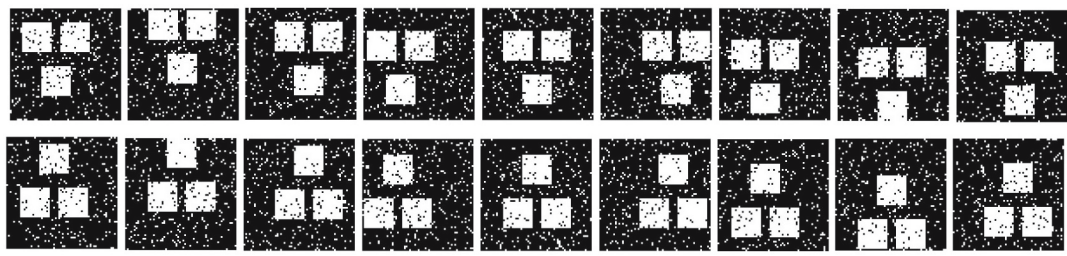

Fig. 2. Plots of an artificial data of two-dimensional images that the proposed method can handle. The top row shows a class of images involving three white squares, and the bottom row shows another class. The two pictures placed in the center of each row are the prototypes of their classes. The rest of the pictures illustrate warps due to left-and-right shifts and up-and-down shifts.

of each sample. To construct the dissimilarity matrix, then we used the proposed correlation coefficient-based DTW method in conjunction with a conventional measurement system, such as Euclidean distance (ED) measuring or Hamming distance (HD) measuring 11. In the notations of ED1D and ED2D, the first two letters refer to the method of measuring the dissimilarity, namely ED measuring; the next two letters refer to the dimensionality; hence, 1D and 2D indicates that to obtain the optimal warping path the correlation coefficient is computed in a one-dimensional way or a two-dimensional way, respectively. In the one-dimensional way, the computation was performed solely with column vectors; in the two-dimensional way, the computation was performed with raw vectors and column vectors.

Because the construction of the dissimilarity matrix is time-consuming, we randomly selected five objects from the AT\&T database and then constructed a $50 \times 50$ dissimilarity matrix instead of a full $400 \times 400$ matrix. This process was repeated 20 times for all objects of the database and a final result was obtained by averaging the results of each repetition 12. On the other hand, to maintain the diversity between the dissimilaritybased classifications, we designed different classifiers, such as the $k$-nearest neighbor classifiers $(k=1,3,5,7)$, the nearest mean classifiers, the support vector classifier, and the regularized normal density-based linear/quadratic classifiers. These classifiers, which were implemented with PRTools 13 , are denoted in the next section as 1-NN, 3-NN, 5-NN, 7-NN, NMC, SVC, RLDC, and RQDC, respectively.

Experimental Results: The classification performance of the DBCs for the artificial data set is first illustrated in graphs. A numerical comparison of the two benchmark

${ }^{11}$ Here, we experimented with only two measures, namely, the ED and HD measuring methods. However, other measuring methods, such as the modified Hausdorff distances, the blurred Euclidean distance, etc., could also be employed. From this perspective, the question "what is the best measure ?" is an interesting issue for further study.

${ }^{12}$ We experimented with the simpler $50 \times 50$ matrix here. However, it should also be mentioned that we can have numerous solutions, depending on the representative selection, the dissimilarity measure, and the design of classifiers in the dissimilarity space. Especially, regarding the dissimilarity measure, it is possible to construct the dissimilarity matrix rapidly by employing a computational technique. From this perspective, we are currently investigating how the experiment with the full $400 \times 400$ matrix can be performed at a high speed.

${ }^{13}$ PRTools is a MATLAB toolbox for pattern recognition (refer to http://www.prtools.org/). 

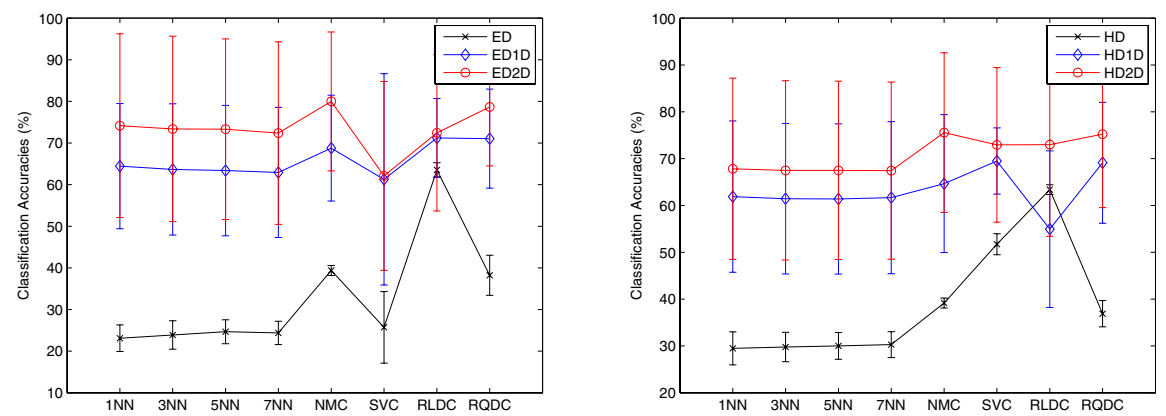

Fig. 3. A comparison of the classification accuracy rates (\%) for the artificial data set: (a) left and (b) right. The dissimilarity matrices of (a) and (b) are obtained with the ED, HD, and their modified measuring methods, respectively.

databases is then made in relation to the conventional methods and the proposed scheme. Figure 3 shows a comparison of the classification accuracy rates (\%) for the artificial data set with different dissimilarity measures.

These figures confirm the possibility of improving the performance of DBCs by effectively measuring the dissimilarity. The improvement can be seen by observing how the classification accuracy rates $(\%)$ change. For example, in Figure 3 , for all the nonparametric classifiers, namely $k$-NN, NMC, and SVC, the classification results of the proposed measuring methods of ED1D and ED2D (which are marked as $\circ$ and $\diamond$ ) are significantly more accurate than those of the conventional ED measuring method (which is indicated as $\times$ ). For the parametric classifiers RLDC and RQDC, the classification results of the proposed methods are also more accurate than those of the conventional methods. The problem of theoretically analyzing this observation remains unresolved.

Table 1. A comparison of the averaged classification accuracy rates (\%) of DBCs for AT\&T and UMIST databases. The details of the table are discussed in the text.

\begin{tabular}{|c|c|c|c|c|c|c|c|c|c|}
\hline Experimental & Experimental & \multicolumn{6}{|c|}{ Non-parametric Classifiers } & \multicolumn{2}{|c|}{ Parametric Ones } \\
\cline { 3 - 9 } Databases & Methods & 1-NN & 3-NN & $5-N N$ & $7-$ NN & NMC & SVC & RLDC & RQDC \\
\hline \hline \multirow{6}{*}{ AT\&T } & ED & 89.00 & 76.80 & 69.70 & 66.70 & 73.90 & 93.60 & $\mathbf{9 5 . 3 0}$ & 84.40 \\
& ED1D & 89.20 & 84.40 & 81.80 & 78.50 & 79.20 & 94.10 & 88.20 & $\mathbf{8 6 . 4 0}$ \\
& ED2D & $\mathbf{9 2 . 8 0}$ & $\mathbf{8 7 . 4 0}$ & $\mathbf{8 5 . 6 0}$ & $\mathbf{8 0 . 6 0}$ & $\mathbf{8 4 . 7 0}$ & $\mathbf{9 6 . 9 0}$ & 76.70 & 84.70 \\
\cline { 2 - 9 } & HD & $\mathbf{9 2 . 6 0}$ & 86.10 & 78.10 & 74.00 & 47.50 & 63.40 & 33.90 & 87.90 \\
& HD1D & 91.80 & 81.10 & 77.10 & 74.00 & 43.70 & 60.90 & 48.50 & $\mathbf{9 2 . 2 0}$ \\
& HD2D & 90.70 & $\mathbf{8 7 . 1 0}$ & $\mathbf{7 9 . 4 0}$ & $\mathbf{7 5 . 9 0}$ & $\mathbf{6 3 . 1 0}$ & $\mathbf{7 4 . 5 0}$ & $\mathbf{6 7 . 5 0}$ & 91.40 \\
\hline \multirow{6}{*}{ UMIST } & ED & 99.27 & 99.07 & 99.00 & 98.33 & 99.07 & $\mathbf{9 9 . 2 7}$ & $\mathbf{9 9 . 2 6}$ & 99.26 \\
& ED1D & $\mathbf{9 9 . 4 0}$ & $\mathbf{9 9 . 3 3}$ & $\mathbf{9 9 . 2 7}$ & $\mathbf{9 9 . 2 7}$ & $\mathbf{9 9 . 2 7}$ & $\mathbf{9 9 . 2 7}$ & $\mathbf{9 9 . 2 6}$ & $\mathbf{9 9 . 5 3}$ \\
& ED2D & 99.33 & $\mathbf{9 9 . 3 3}$ & $\mathbf{9 9 . 2 7}$ & $\mathbf{9 9 . 2 7}$ & $\mathbf{9 9 . 2 7}$ & $\mathbf{9 9 . 2 7}$ & 98.33 & 99.33 \\
\cline { 2 - 9 } & HD & 99.93 & $\mathbf{1 0 0}$ & $\mathbf{1 0 0}$ & 99.8 & 87.53 & $\mathbf{9 9 . 0 0}$ & 90.00 & 99.13 \\
& HD1D & $\mathbf{1 0 0}$ & $\mathbf{1 0 0}$ & $\mathbf{1 0 0}$ & $\mathbf{1 0 0}$ & 91.33 & $\mathbf{9 9 . 0 0}$ & 93.53 & 99.20 \\
& HD2D & $\mathbf{1 0 0}$ & $\mathbf{1 0 0}$ & $\mathbf{1 0 0}$ & $\mathbf{1 0 0}$ & $\mathbf{9 2 . 1 3}$ & $\mathbf{9 9 . 0 0}$ & $\mathbf{9 6 . 3 3}$ & $\mathbf{1 0 0}$ \\
\hline
\end{tabular}


To further investigate the advantage of using the proposed method, we conducted the following experiments for the real benchmark databases, namely AT\&T and UMIST. First, the 50 facial images of five individuals were randomly selected from AT\&T (and UMIST). Next, for the images, we evaluated the classification performance of the DBCs, which were designed in dissimilarity matrices constructed with conventional measures (of ED and HD) and the proposed methods (of ED1D, ED2D, HD1D and HD2D). After repeating the above two steps 20 times, we averaged the performances. (The best results of these evaluations are printed in bold in Table 1) In comparing the conventional and new schemes, Table 1 shows a comparison of the averaged classification accuracy rates $(\%)$ for the facial images of AT\&T and UMIST. We observed the same characteristics in Table 1 as in Figure 3 (see the bold-faced numbers). These results confirm that the classification performance of almost all classifiers was improved with the ED1D method and further improved with the ED2D method.

In review, it is not easy to crown one particular measuring method with superiority over the others in terms of solving the dissimilarity measuring problem. However, in terms of classification accuracies, the proposed measuring method is clearly more useful for simple pixel-based measurements, such as ED and HD measuring.

\section{Conclusions}

In our efforts to optimize DBCs, we used the DTW dynamic programming technique. To construct a dissimilarity matrix of DBCs, we measured the dissimilarity directly from the input images with one-to-many (or many-to-one) mapping as well as oneto-one mapping. This method overcomes the problems caused by variations in facial directions and sizes. Furthermore, instead of measuring the dissimilarity distances on the basis of the entire image, we used the correlation coefficient-based method where the entire image region is divided into several subregions according to their distances. The proposed method was tested on an artificial image data set and two well-known benchmark databases, and the results were compared with the results of conventional methods. The experimental results demonstrate that the proposed method is better than conventional methods in terms of classification accuracy. Although we have shown that DBCs can be optimized with our proposed measuring scheme, many tasks remain open. One of them is to improve the classification efficiency by combining the optimized classifiers in the dissimilarity space. Our aim is to conduct further research on this subject in the future.

\section{References}

1. Jain, A.K., Duin, R.P.W., Mao, J.: Statistical pattern recognition: A review. IEEE Trans. Pattern Anal. and Machine Intell. PAMI 22(1), 4-37 (2000)

2. Pekalska, E., Duin, R.P.W.: The Dissimilarity Representation for Pattern Recognition: Foundations and Applications. World Scientific Publishing, Singapore (2005)

3. Pekalska, E., Duin, R.P.W., Paclik, P.: Prototype selection for dissimilarity-based classifiers. Pattern Recognition 39, 189-208 (2006)

4. Kim, S.-W., Oommen, B.J.: On using prototype reduction schemes to optimize dissimilaritybased classification. Pattern Recognition 40, 2946-2957 (2007) 
5. Belhumeour, P.N., Hespanha, J.P., Kriegman, D.J.: Eigenfaces vs. Fisherfaces: Recognition using class specific linear projection. IEEE Trans. Pattern Anal. and Machine Intell., PAMI 19(7), 711-720 (2007)

6. Zhou, W., Chellappa, R., Rosenfeld, A., Phillips, P.J.: Face recognition: a literature survey. ACM Compt. Surveys 35(4), 399-458 (2003)

7. Lozano, M., Sotoca, J.M., Sanchez, J.S., Pla, F., Pekalska, E., Duin, R.P.W.: Experimental study on prototype optimisation algorithms for prototype-based classification in vector spaces. Pattern Recognition 39, 1827-1838 (2006)

8. Orezco-Alzate, M., Duin, R.P.W., Castellanos-Dominguez, C.G.: Generalizing dissimilarity representations using feature lines. In: Rueda, L., Mery, D., Kittler, J. (eds.) CIARP 2007. LNCS, vol. 4756, pp. 370-379. Springer, Heidelberg (2007)

9. Paredes, R., Vidal, E.: A class-dependent weighted dissimilarity measure for neareast neighbor classification problem. Pattern Recognition Letters 21(12), 1027-1036 (2000)

10. Wang, J., Neskovic, P., Cooper, L.N.: Improving neareast neighbor rule with a simple adaptive distance measure. Pattern Recognition Letters 28(2), 207-213 (2007)

11. Kim, S.-W.: Optimizing dissimilarity-based classifiers using a newly modified Hausdorff distance. In: Hoffmann, A., Kang, B.-h., Richards, D., Tsumoto, S. (eds.) PKAW 2006. LNCS, vol. 4303, pp. 177-186. Springer, Heidelberg (2006)

12. Kim, S.-W., Duin, R.P.W.: On combining dissimilarity-based classifiers to solve the small sample size problem for appearance-based face recognition. In: Kobti, Z., Wu, D. (eds.) Canadian AI 2007. LNCS, vol. 4509, pp. 110-121. Springer, Heidelberg (2007)

13. Sakoe, H., Chiba, S.: A dynamic programming algorithm optimization for spoken word recognition. IEEE Trans. Acoust. Speech Signal Process. 26(1), 43-49 (1978)

14. Rabiner, L., Jung, B.H.: Fundamentals of Speech Recognition. Prentice Hall PTR, New Jersey (1993)

15. Ratan, A.L., Grimson, W.E.L., Wells, W.M.: Object detection and localization by dynamic template warping. International Journal of Computer Vision 36(2), 131-147 (2000)

16. Sahbi, H., Boujemaa, N.: Robust face recognition using dynamic space warping. In: Tistarelli, M., Bigun, J., Jain, A.K. (eds.) ECCV 2002. LNCS, vol. 2359, pp. 121-132. Springer, Heidelberg (2002)

17. Qiao, Y., Yasuhara, M.: Affine invariant dynamic time warping and its application to online rotated handwriting recognition. In: Proceedings of International Conference on Pattern Recognition, ICPR 2006, vol. 2, pp. 905-908. IEEE Computer Society, Los Alamitos (2006) 